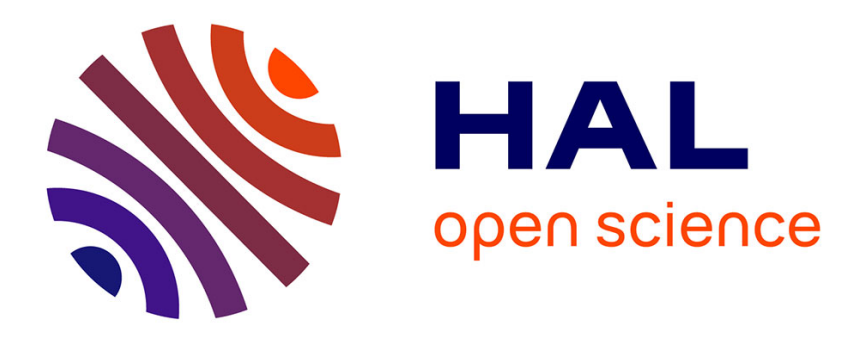

\title{
Adapting the measurement methods to the different purposes
}

\author{
Paul Robin, Mélynda Hassouna
}

\section{To cite this version:}

Paul Robin, Mélynda Hassouna. Adapting the measurement methods to the different purposes. ACS Fall 2020 Virtual Meeting - Symposium on Animal agriculture emission measurement technologies, Kyoung S Ro (USDA); Mélynda Hassouna (INRAE), Aug 2020, San Fransisco, United States. 10.1021/scimeetings.0c07421 . hal-03324203

\section{HAL Id: hal-03324203 \\ https://hal.inrae.fr/hal-03324203}

Submitted on 23 Aug 2021

HAL is a multi-disciplinary open access archive for the deposit and dissemination of scientific research documents, whether they are published or not. The documents may come from teaching and research institutions in France or abroad, or from public or private research centers.
L'archive ouverte pluridisciplinaire HAL, est destinée au dépôt et à la diffusion de documents scientifiques de niveau recherche, publiés ou non, émanant des établissements d'enseignement et de recherche français ou étrangers, des laboratoires publics ou privés. 


\section{INRAC}

$>$ Adapting the measurement methods to the different purposes

Mélynda Hassouna, Paul Robin

INRAe-UMR SAS-FRANCE

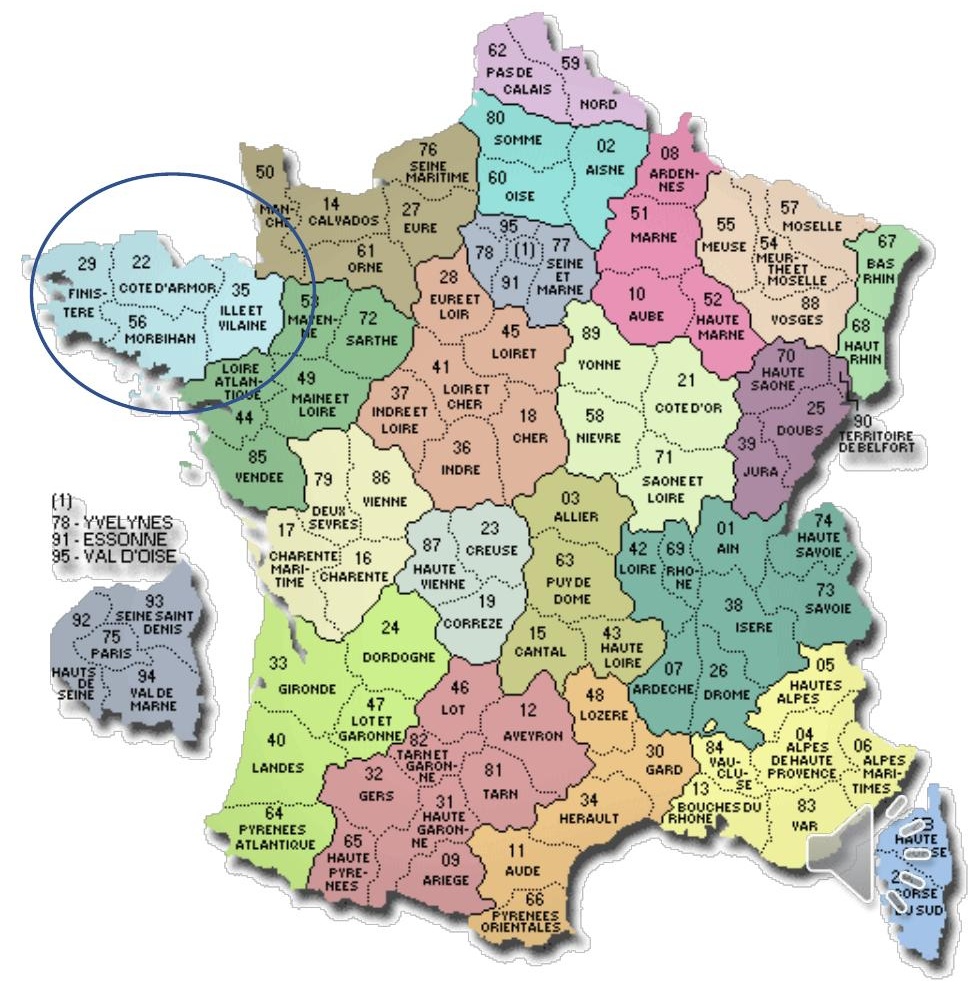



Different gases from various sources
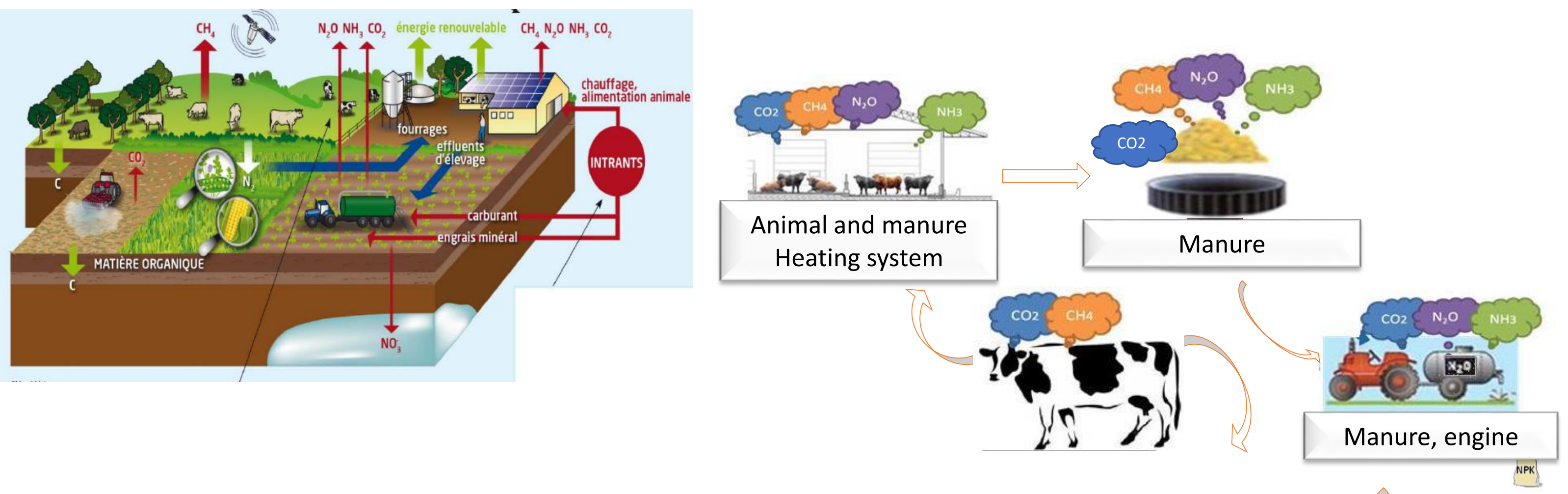


\section{> Specificities of agricultural emissions}
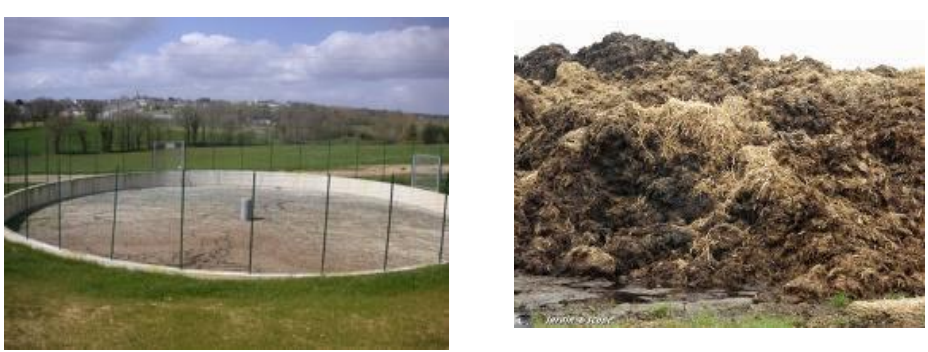

FUGITIVE

Diffuse
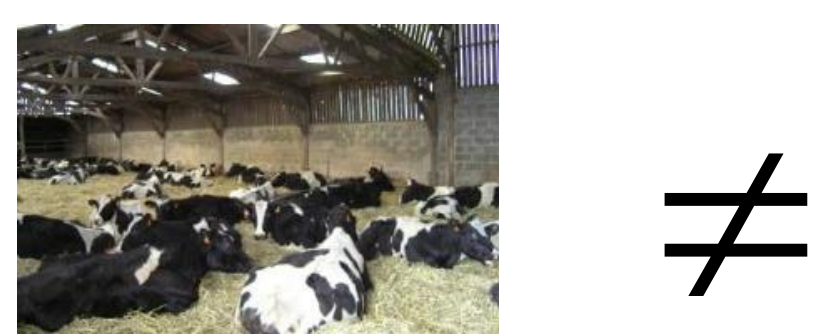

Animal species, genetics, climate, manure management, farmers practices....

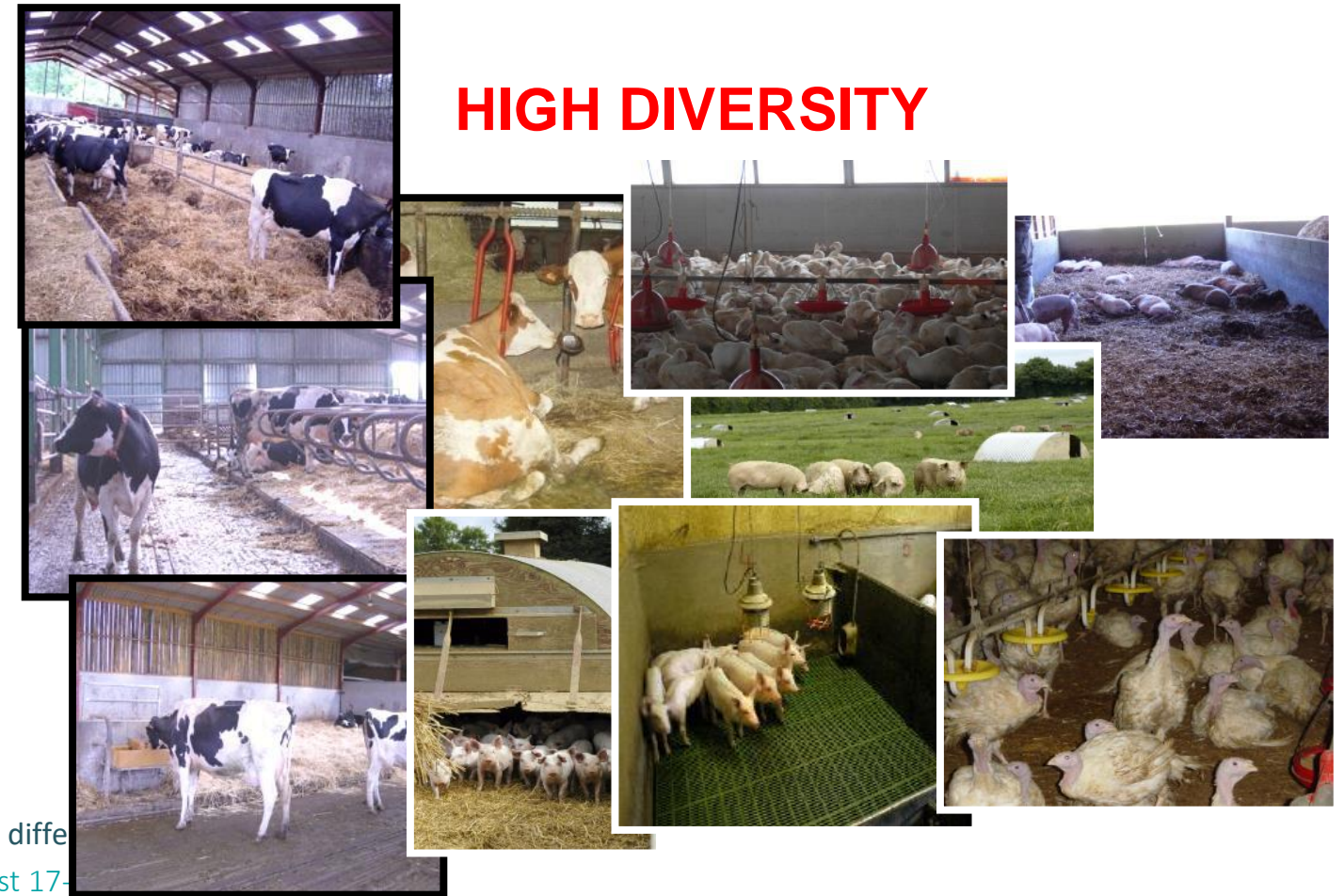

CHANELED 
$>$ Why quantifying agricultural emissions?

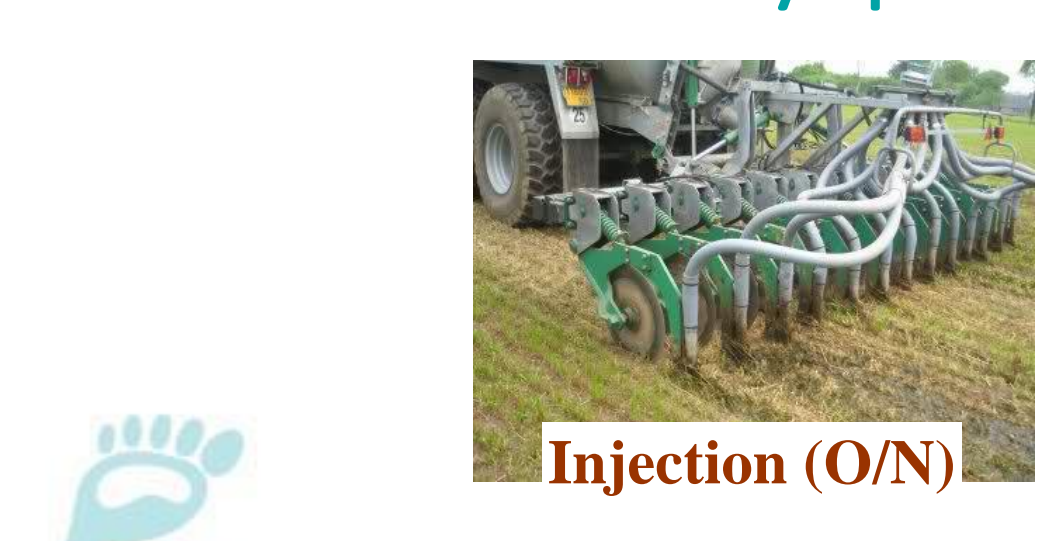
$\rightarrow$ MITIGATION

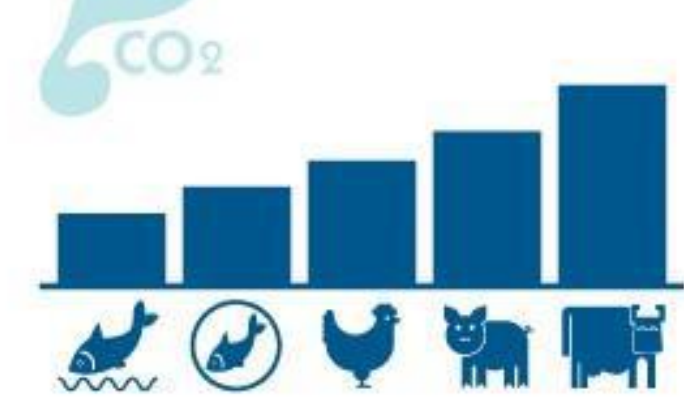

Certification of mitigation techniques and emission levels (regulation) GHG 1990-2050, France
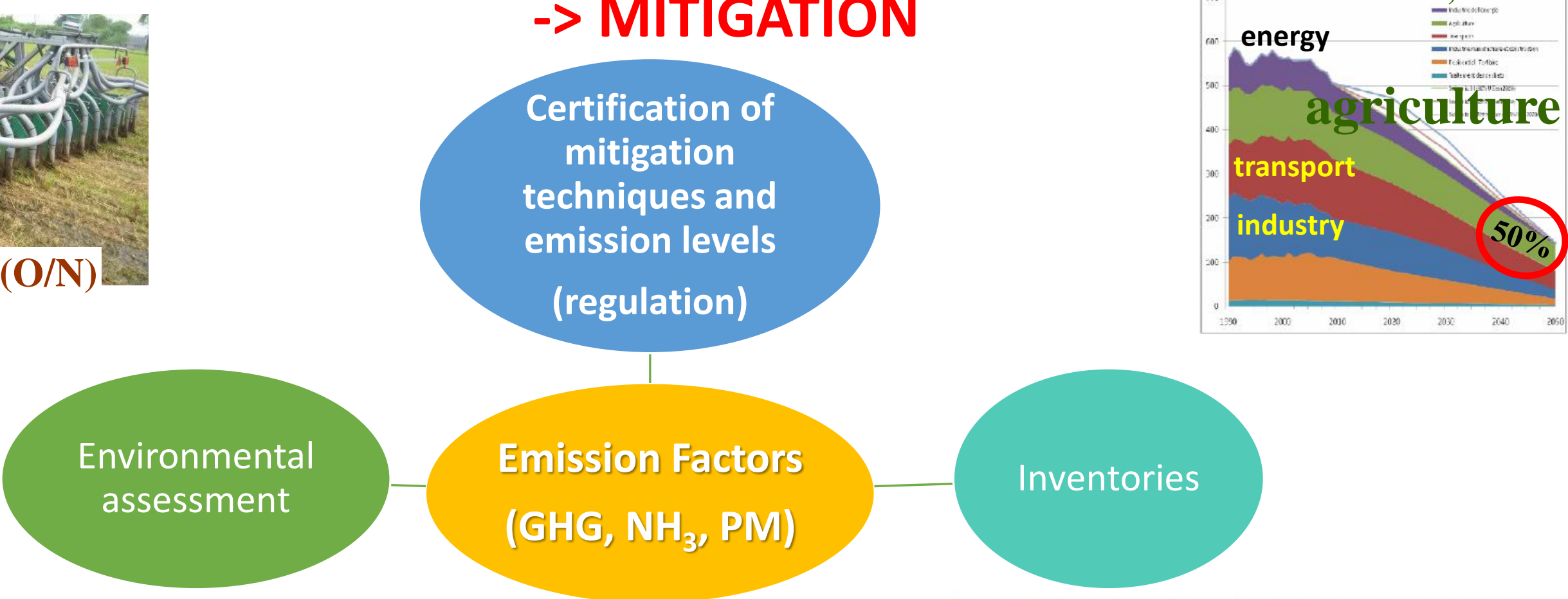

Emission Factors (GHG, NH $H_{3,}$, PMI)

\section{Inventories}

Figure 1. Ammonia emissions, EU-28, 2013 (\% of total ammonia emissions).
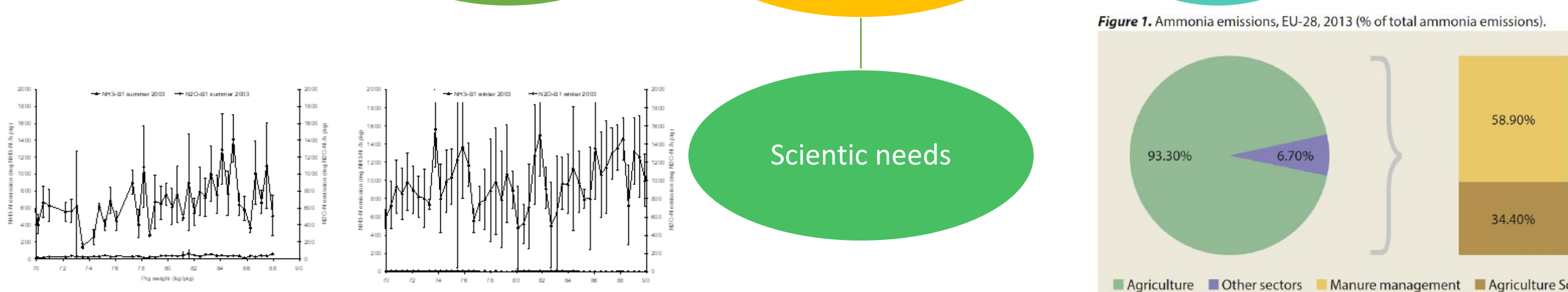

agriculture $=$ Other sectors Manure management $=$ Agriculture Soils INRAC 


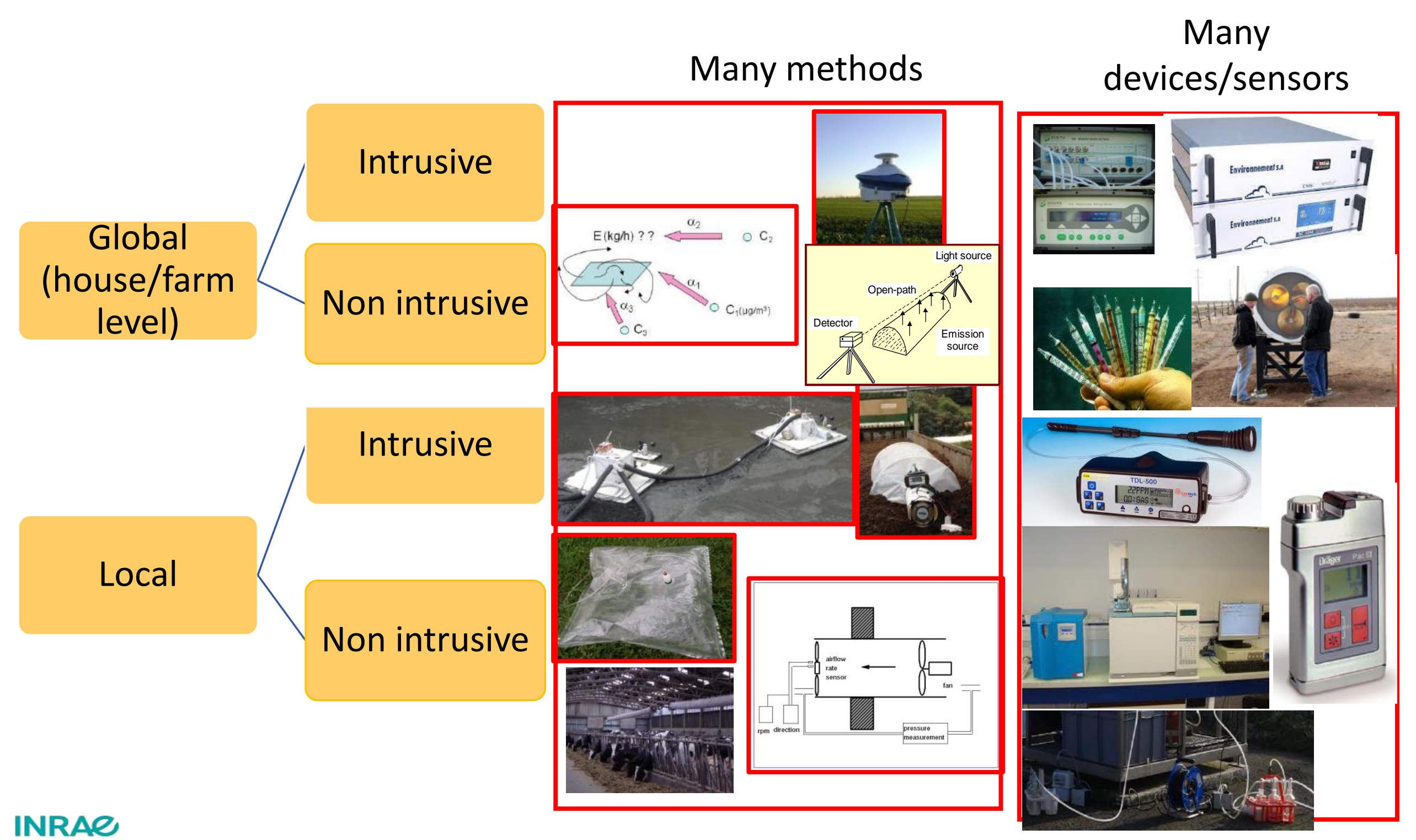


$>$ Different kind of measurement objectives with various constraints

$\mathbf{R}$

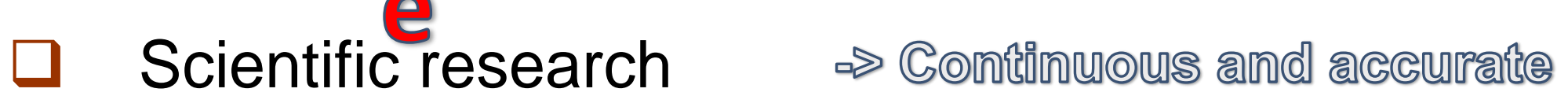

I

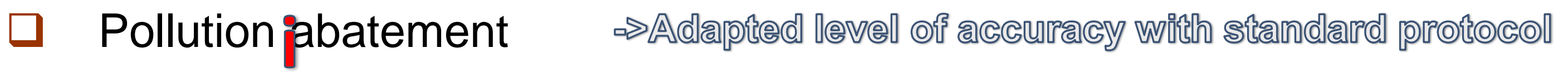

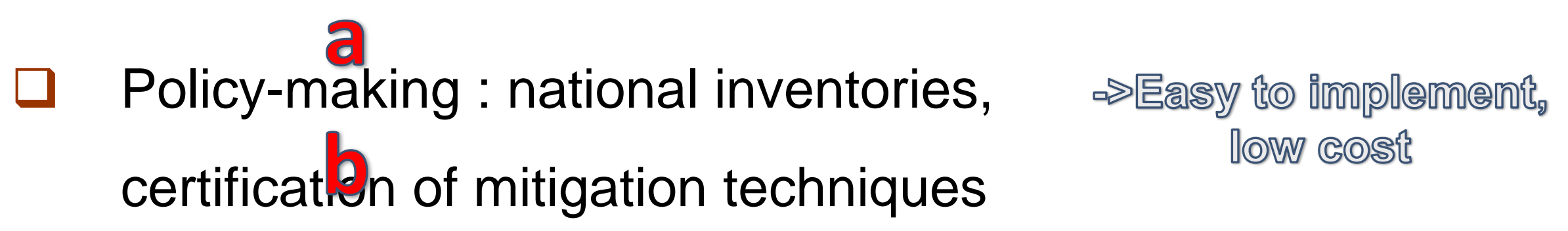

I 


\section{Different kinds of objectives with many possible constraints}

\section{INRAC}

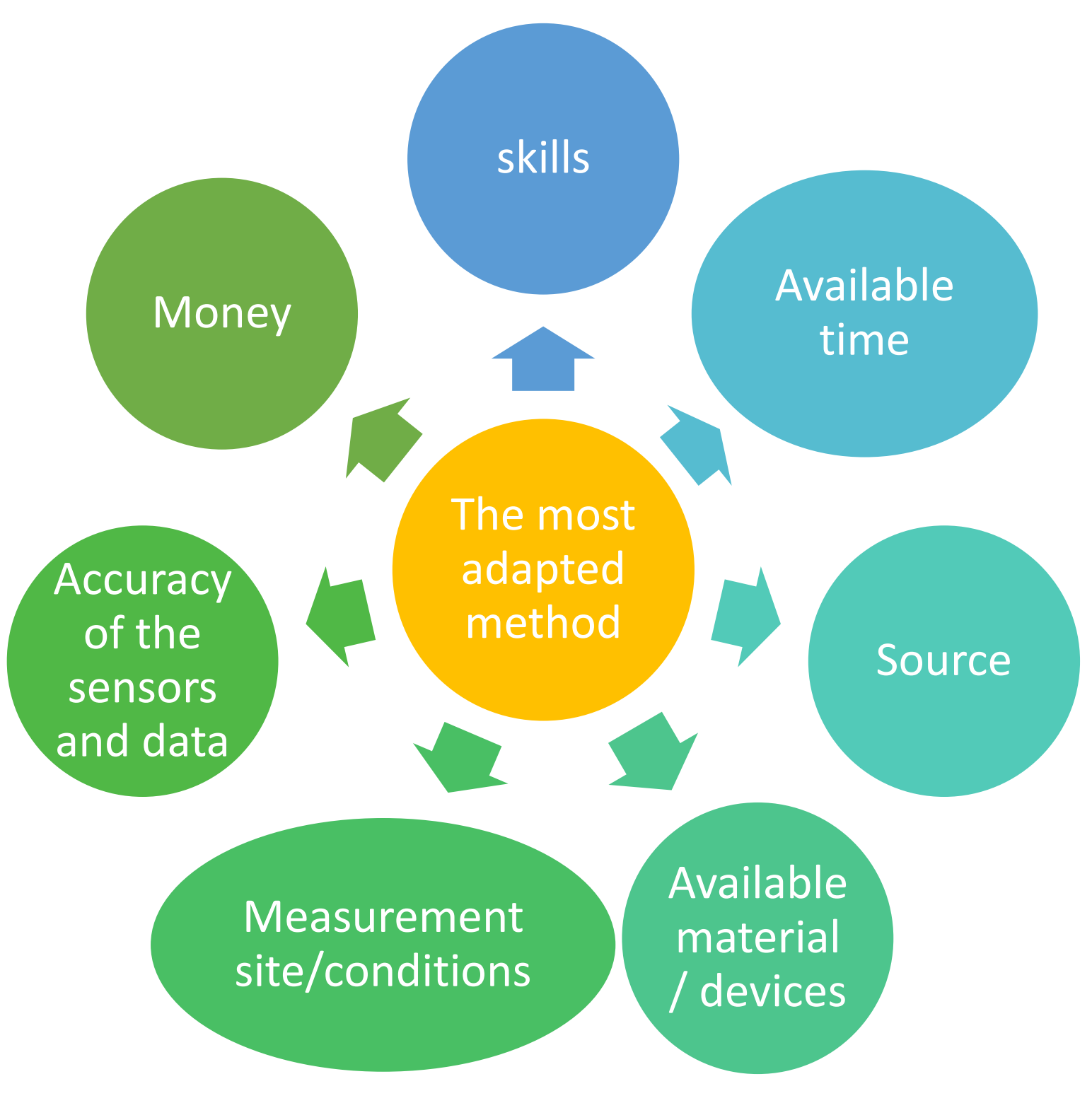




\section{$>3$ kinds of measurement methodology}

\begin{tabular}{l} 
Reference metho \\
$\begin{array}{l}\text { Continuous } \\
\text { measurement }\end{array}$ \\
$\begin{array}{l}\text { Studies of } \\
\text { emitting } \\
\text { processes }\end{array}$ \\
\hline Scientists
\end{tabular}

\section{Simplified Methods \\ Intermittent measurement + models}

Low-cost methods

Efficiency of mitigation options and environmental certification

\section{Emission-factor acquisition}

Scientists, agricultural engineers, monitoring and certification offices
Control methods

$$
\begin{aligned}
& \text { Intermittent } \\
& \text { measurement }
\end{aligned}
$$

\section{Low-cost methods}

\section{Emission levels} checking

Agricultural engineers, monitoring and certification offices and livestock technicians, farmers 


\section{$>$ Reference methods}

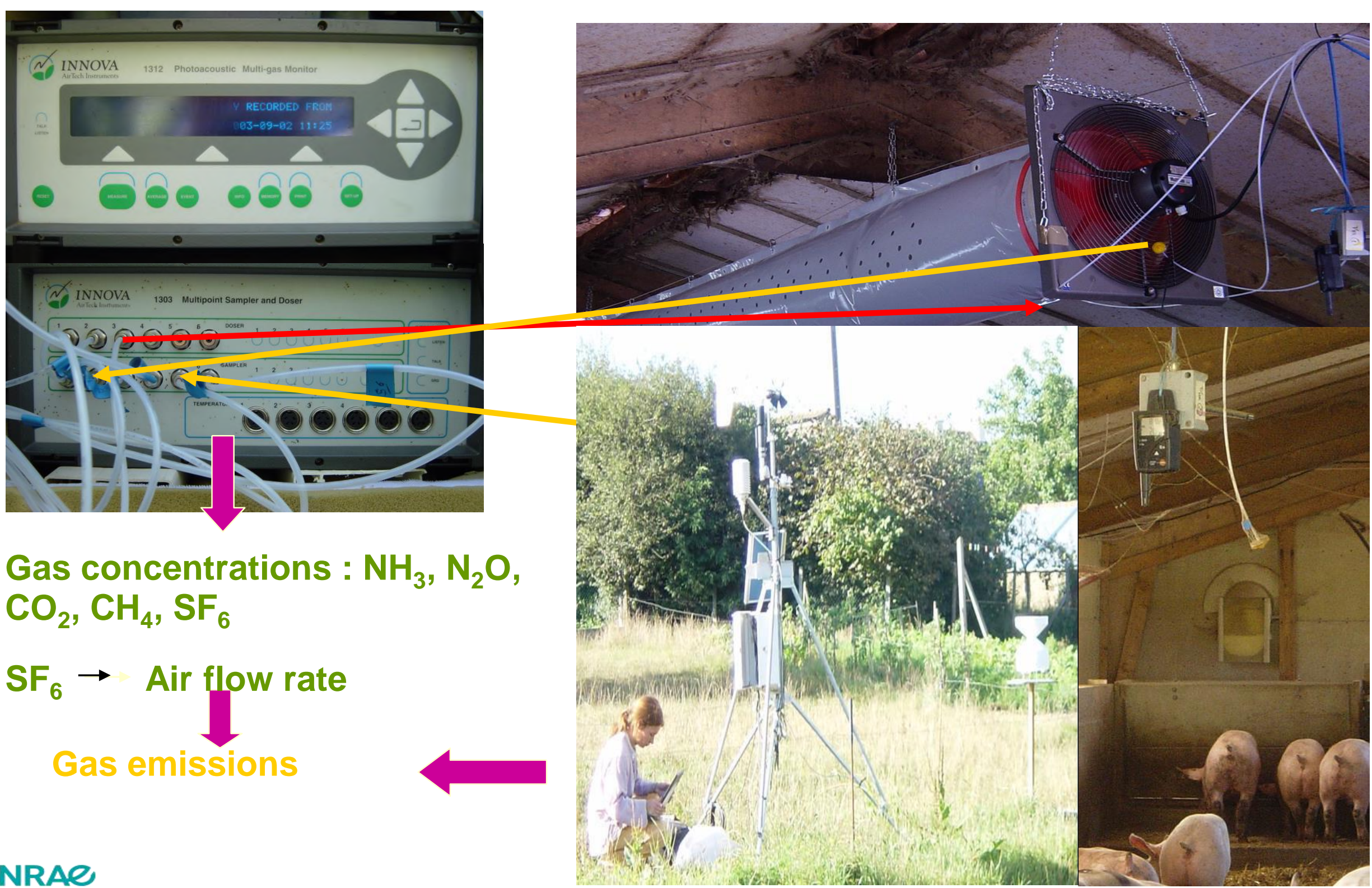




\section{$>$ Reference methods}

Continuous measurement of ER

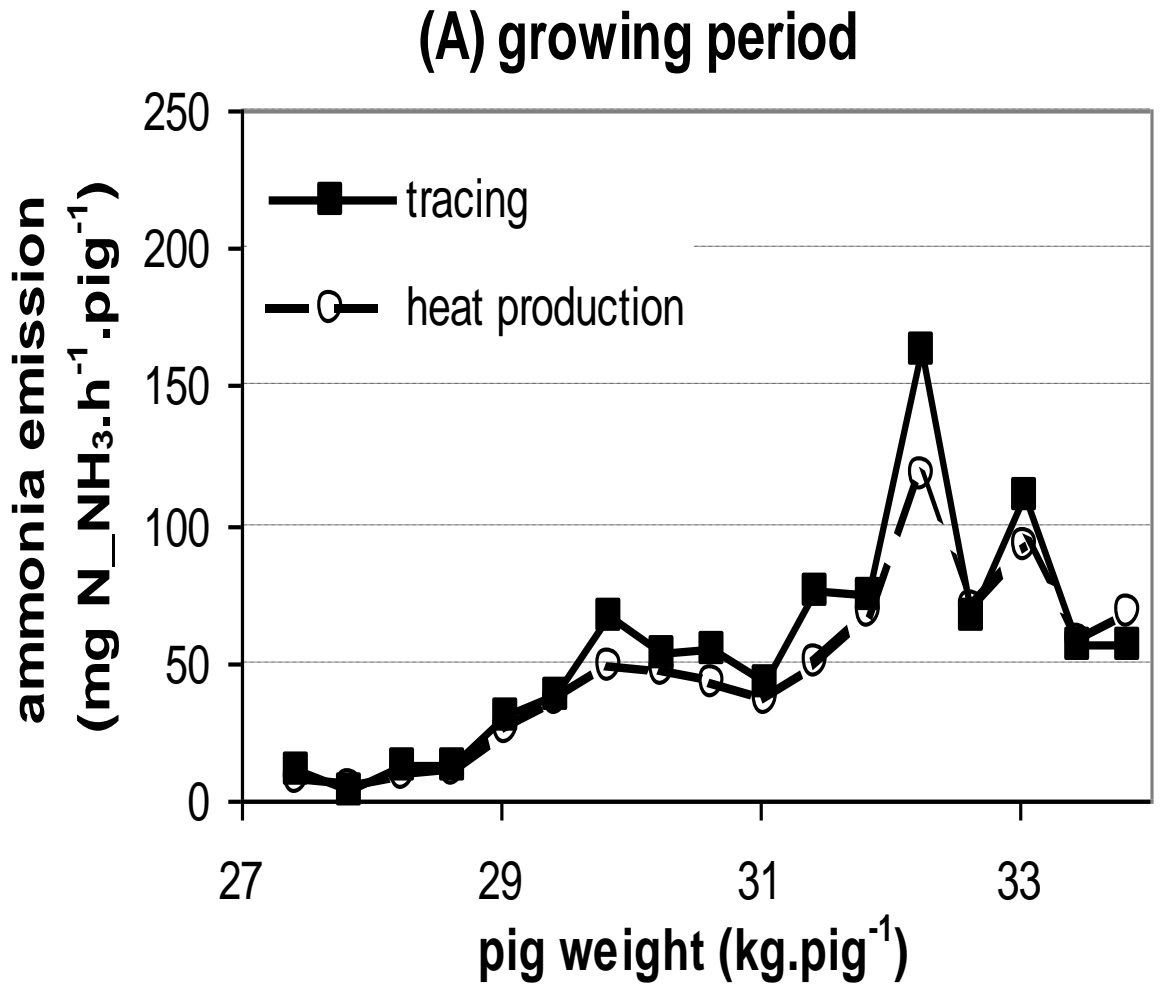

Implementation in experimental facilities

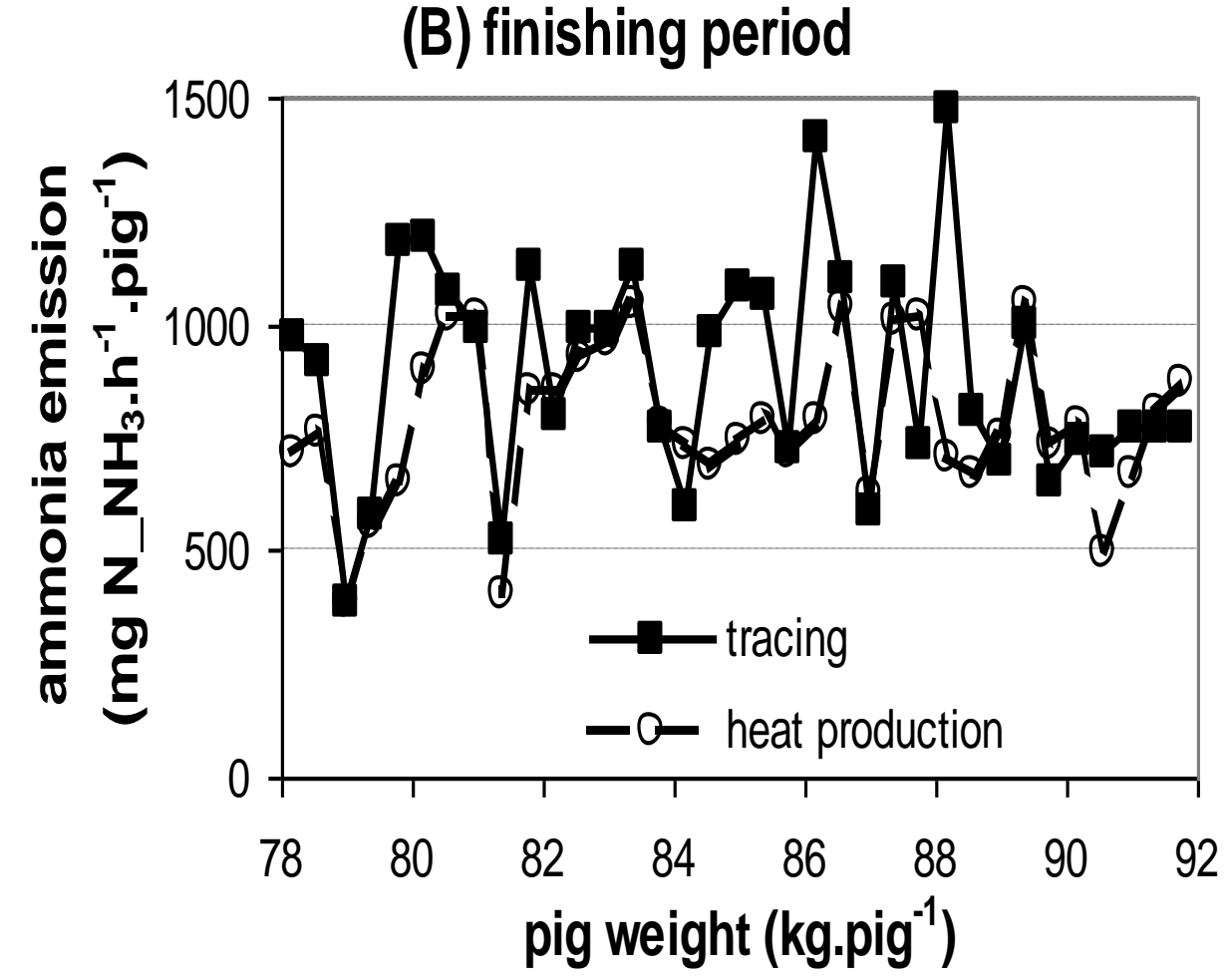

Implementation in commercial conditions

\section{Accuracv}

\section{INRAC}

Adapting the measurement methods to the different purposes 
$>$ Simplified methods

$>$ Example : Ratio of concentration gradients and Carbon mass balance

Spot

measurement of

gas

concentrations

Livestock Data

$$
\begin{gathered}
\text { Convective } \\
\text { transport }
\end{gathered} \longrightarrow \begin{aligned}
& \mathrm{E}_{\mathrm{C}-\mathrm{CO} 2} / \mathrm{E}_{\mathrm{C}-\mathrm{CH} 4}=\operatorname{grad}_{\mathrm{C}-\mathrm{CO} 2} / \operatorname{grad}_{\mathrm{C}-\mathrm{CH} 4} \\
& \mathrm{E}_{\mathrm{N}-\mathrm{NH} 3} / \mathrm{E}_{\mathrm{C}-\mathrm{CO} 2}=\operatorname{grad}_{\mathrm{N}-\mathrm{NH} 3} / \operatorname{grad}_{\mathrm{C}-\mathrm{CO} 2}
\end{aligned}
$$

$$
\begin{gathered}
\text { Mass } \\
\text { budget }
\end{gathered} \underset{\mathrm{C}_{\text {Loss }}=\mathrm{C}_{\text {inputs }} \text { (feed, pigs) }-\mathrm{C}_{\text {outputs }} \text { (manure, pigs) }}{ }
$$

$\rightarrow \mathrm{E}_{\mathrm{C}-\mathrm{CO} 2}=\mathrm{C}_{\text {Loss }} /\left[1+\left(\operatorname{grad}_{\mathrm{C}-\mathrm{CH} 4} / \operatorname{grad}_{\mathrm{C}-\mathrm{CO} 2}\right)\right.$

Emission

estimates

$\rightarrow \mathrm{E}_{\mathrm{C}-\mathrm{CH} 4}=\mathrm{E}_{\mathrm{C}-\mathrm{CO} 2} *\left(\operatorname{grad}_{\mathrm{C}-\mathrm{CH} 4} / \operatorname{grad}_{\mathrm{C}-\mathrm{CO} 2}\right)$

$\rightarrow \mathrm{E}_{\mathrm{N}-\mathrm{NH} 3}=\mathrm{E}_{\mathrm{C}-\mathrm{CO} 2} *\left(\operatorname{grad}_{\mathrm{N}-\mathrm{NH} 3} / \operatorname{grad}_{\mathrm{C}-\mathrm{CO} 2}\right)$

$\rightarrow \mathrm{E}_{\mathrm{N}-\mathrm{N} 2 \mathrm{O}}=\mathrm{E}_{\mathrm{C}-\mathrm{CO} 2} *\left(\operatorname{grad}_{\mathrm{N}-\mathrm{N} 2 \mathrm{O}} / \operatorname{grad}_{\mathrm{C}-\mathrm{CO} 2}\right)$ 
> Simplified method
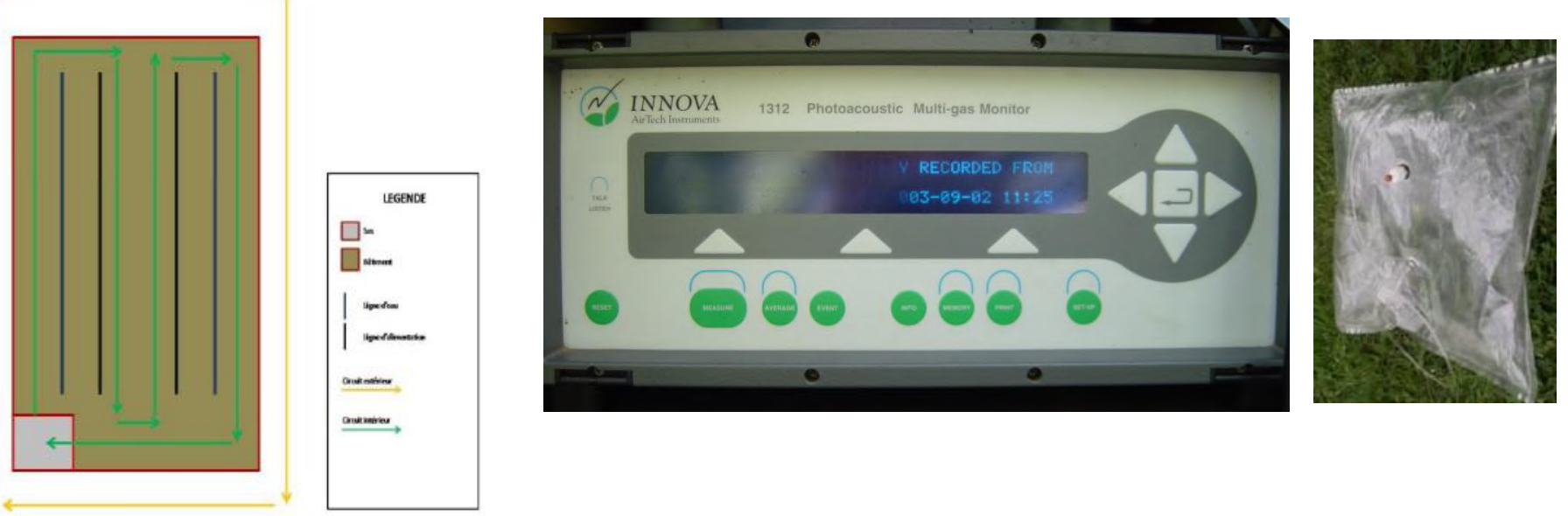

Implemented in $\mathbf{4 0}$ commercial pig houses to quantify EF ADEME Still goes on

+ Zootechnical data
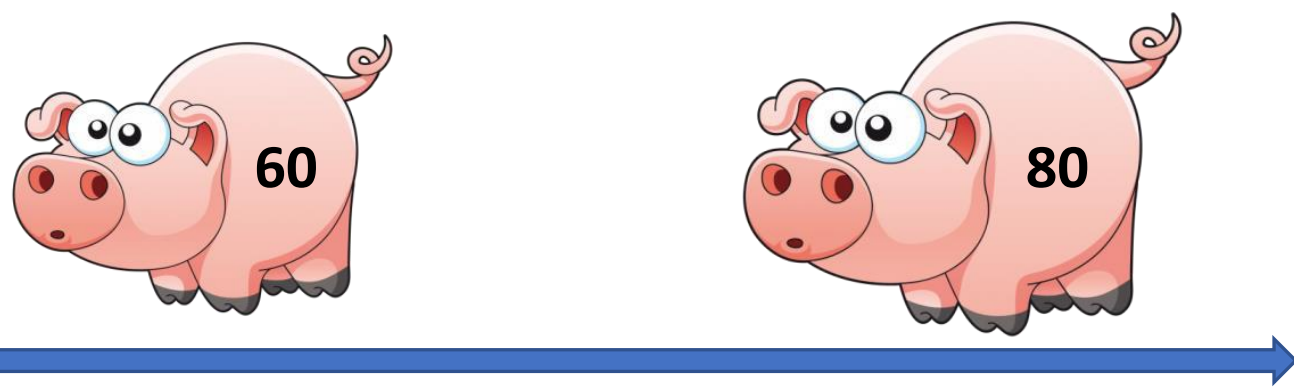


\section{> Simplified method}

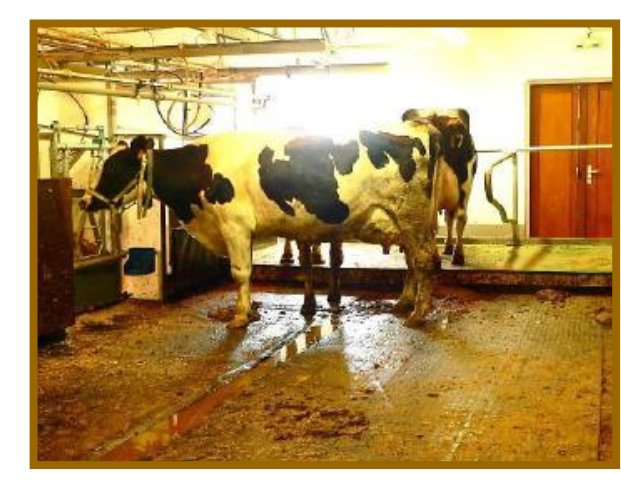

Dairy barns
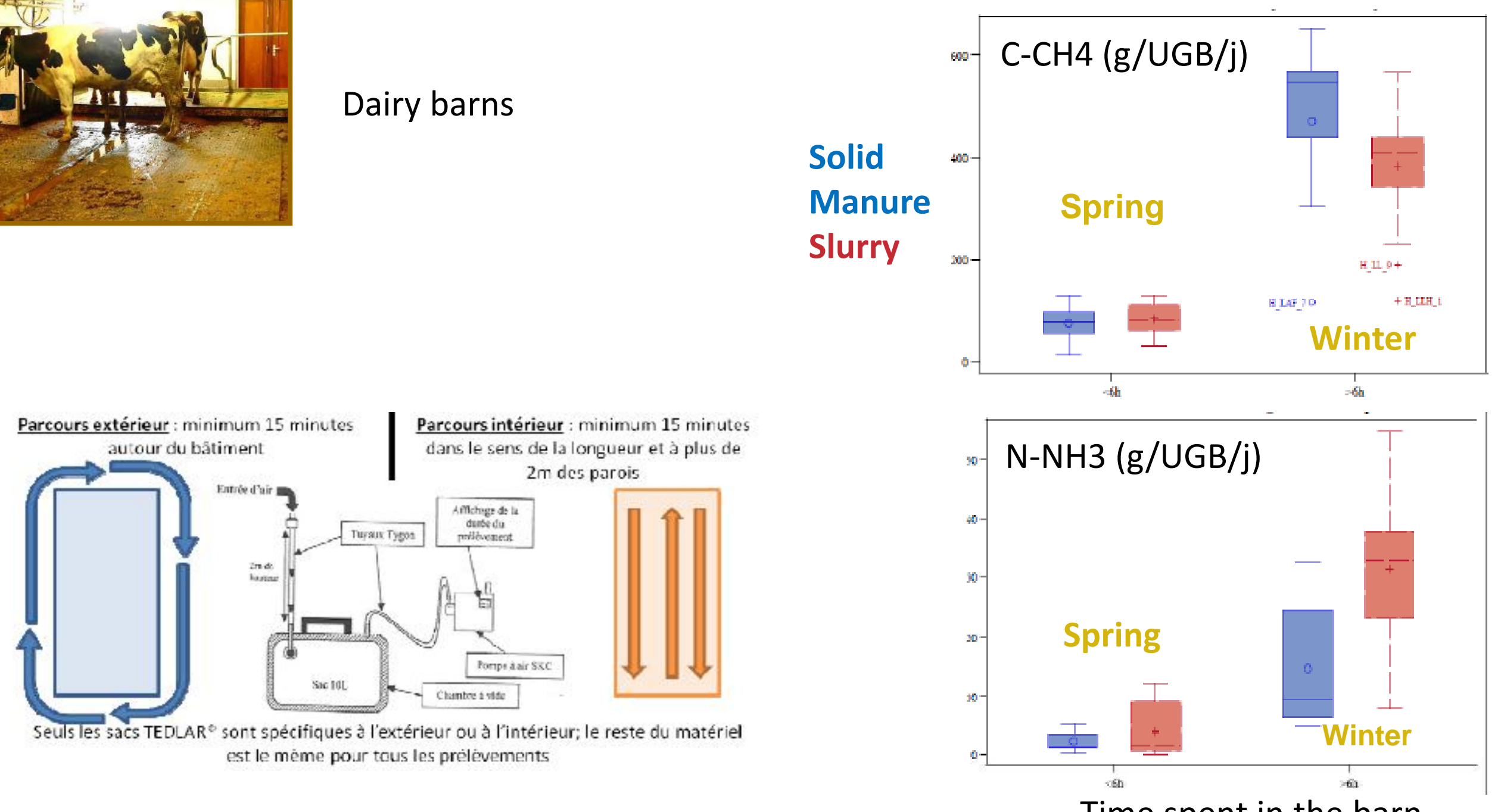

INRAC

Adapting the measurement methods to the different purposes

ACS Fall 2020 Virtual Meeting \& Expo, August 17-20, 2020/ Hassouna

Time spent in the barn 


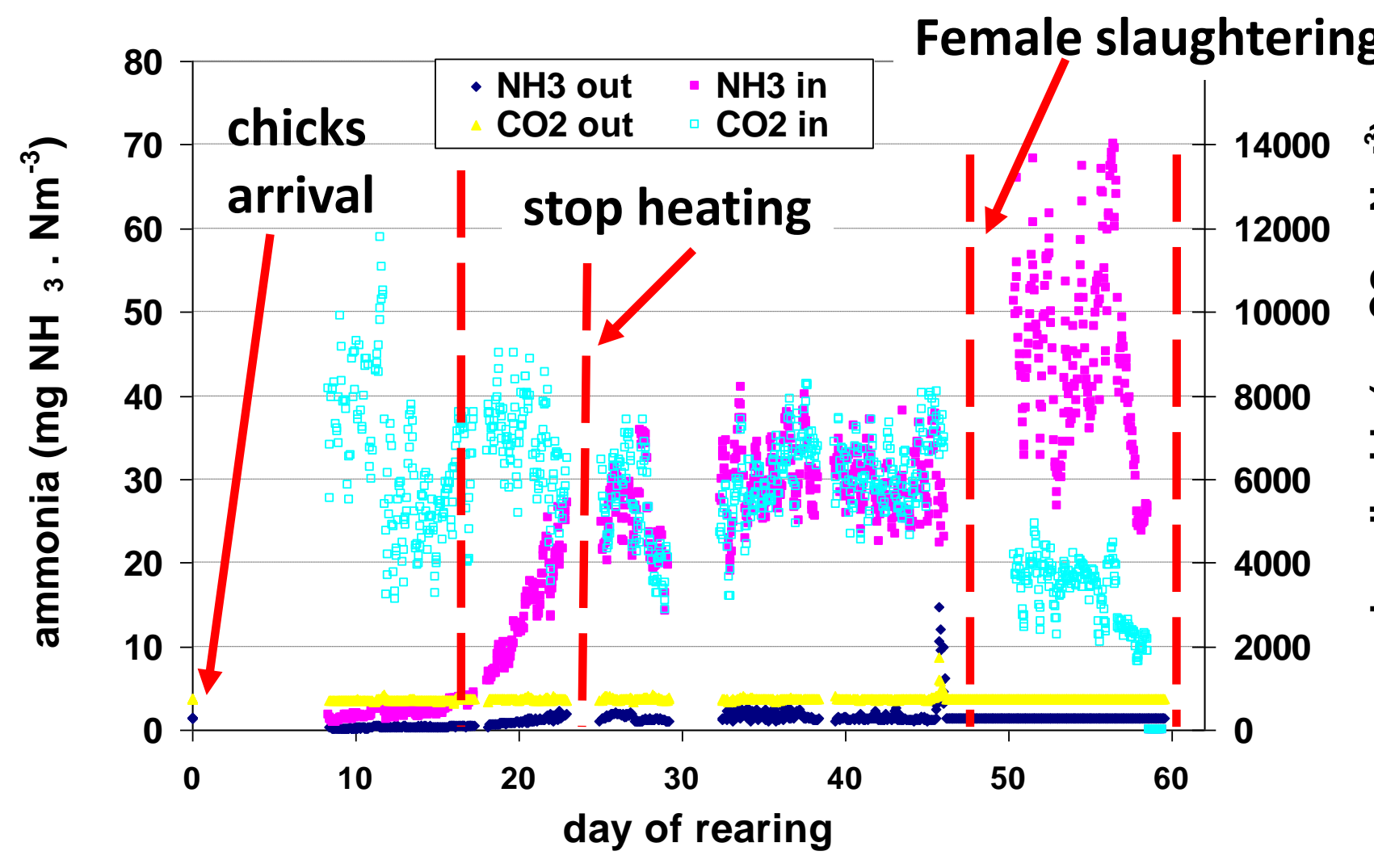

- broiler batch, December 2008, France

- $\mathrm{CO}_{2}$ and $\mathrm{NH}_{3} \pm$ proportional per period - $\mathrm{CO}_{2}$ and $\mathrm{NH}_{3}$ depend on growth, stocking density, manure management

\section{$\operatorname{grad} \mathrm{CO}_{2} /$ grad $\mathrm{NH}_{3}$ characterizes one period}


What are the future challenges

- Keep on working on the validation of simplified methods and control methods

- Standard protocols for measurement but also for uncertainty assessment and sensors calibration

- Guidelines for data reporting

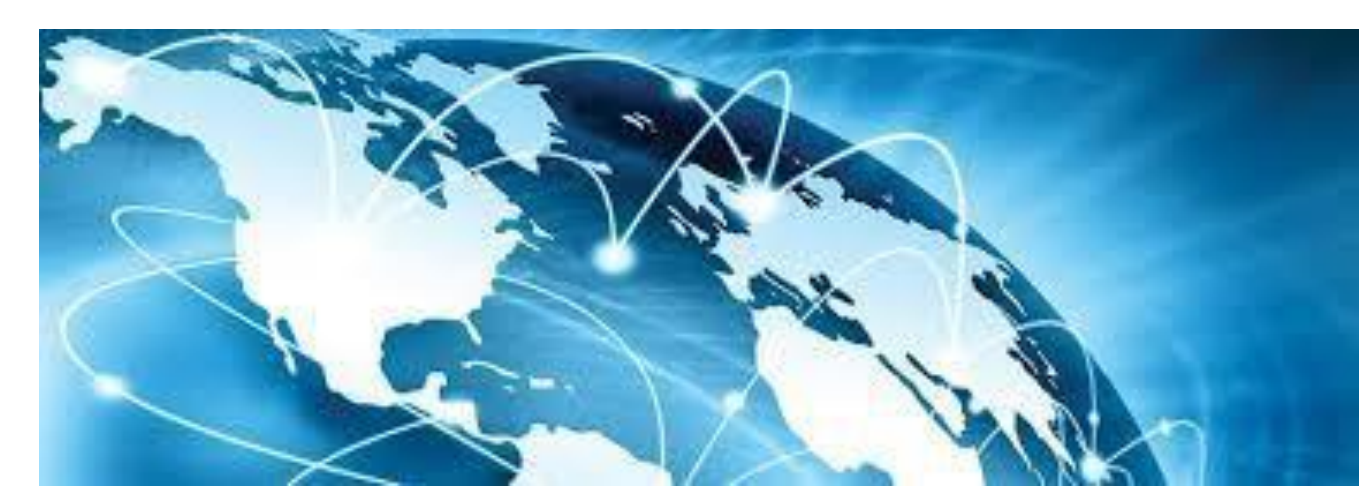

To be more efficient, we should join our forces in an international network on measurement of agricultural gas emissions 


\section{Protocols :}

https://www6.inra.fr/animal_emissions/ADEME-Metrologie

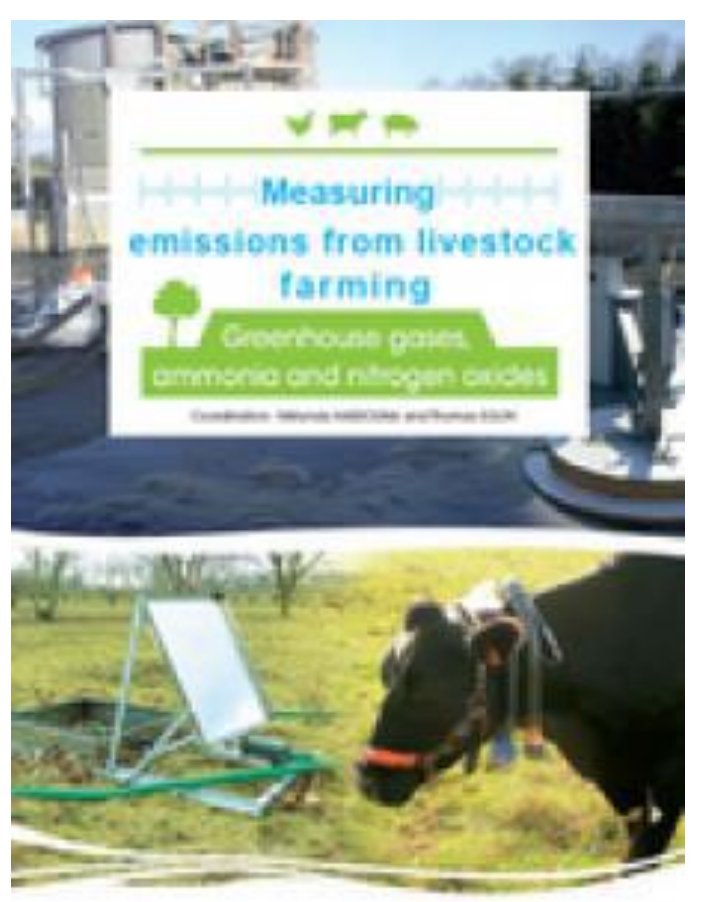

Book Free download

https://www6.inra.fr/animal_emissions_eng/News/Measuringgaseous-emissions-from-animal-farms 
Thanks for your attention and your questions !

\section{Contact :}

melynda.hassouna@inrae.fr

Paul.robin@inrae.fr
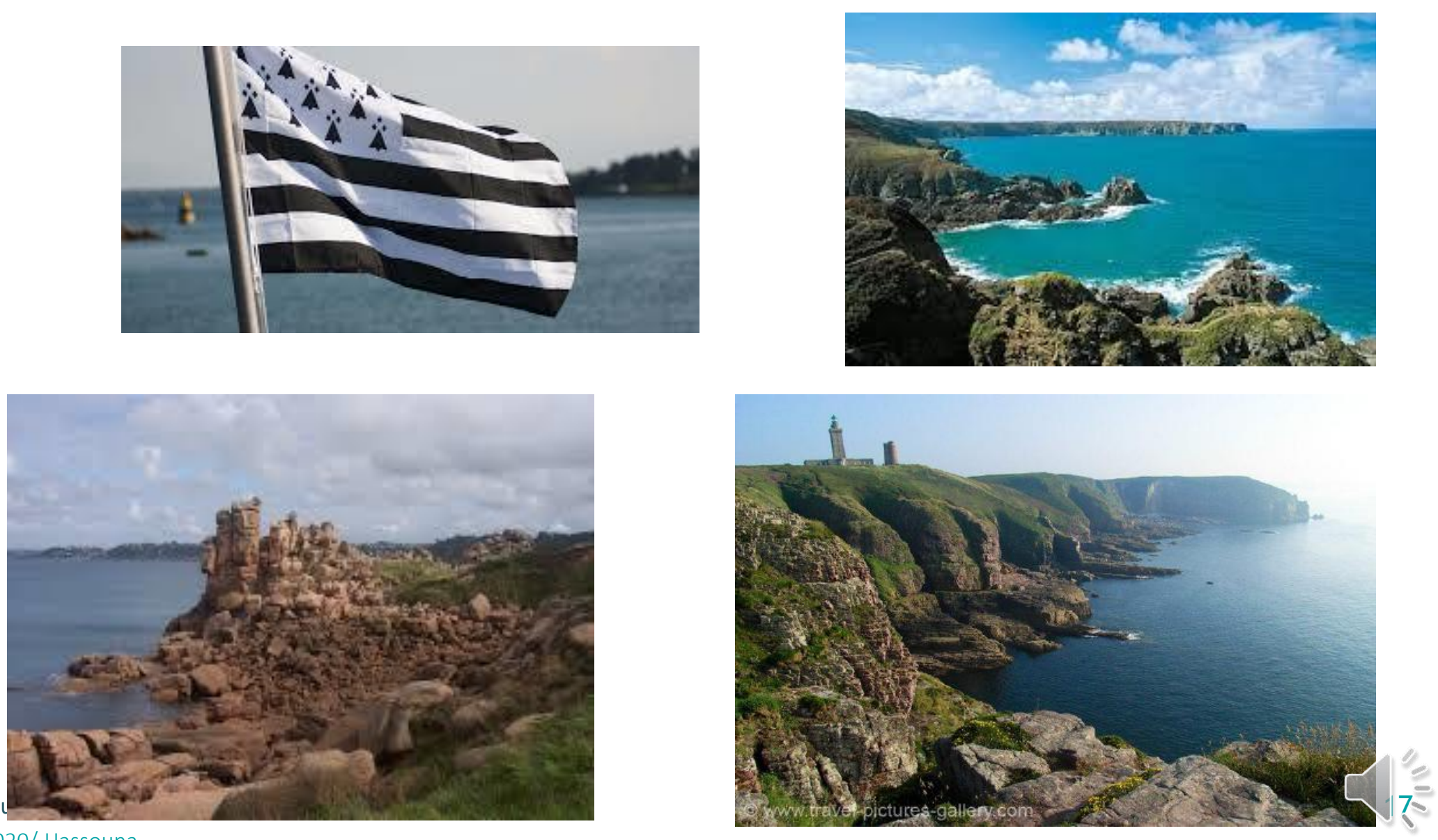